\title{
The sensitivity analysis of scaling criteria in gas hydrate reservoir physical simulation
}

\author{
Yuhu Bai ${ }^{\mathrm{a}, *}$, Hao Yang ${ }^{\mathrm{b}}$, Yan Du ${ }^{\mathrm{c}}$, Ying Zhao ${ }^{\mathrm{d}}$ \\ ${ }^{a}$ China National Offshore Oil Corporation, Research Institute, Beijing 100027, China \\ ${ }^{\mathrm{b}}$ Xinxing Heavy Industries Co., Ltd., Beijing 100070, China \\ ${ }^{\mathrm{c}}$ Zhejiang Ocean University, Zhoushan, Zhejiang 31600, China \\ ${ }^{\mathrm{d}}$ Institute of Mechanics, Chinese Academy of Sciences, Beijing 100190, China
}

\section{A R T I C L E I N F O}

\section{Article history:}

Received 14 October 2009

Received in revised form 17 November 2012

Accepted 17 November 2012

Available online 20 December 2012

\section{Keywords:}

Gas hydrate reservoir

Depressurization

Physical simulation

Sensitivity analysis

\begin{abstract}
A B S T R A C T
A numerical approach of sensitivity analysis of dimensionless parameters in gas hydrate reservoir physical simulation is proposed by analyzing the sensitivity factor defined as the relative variation ration of a target function with respect to the relative variation of dimensionless parameter. With this approach, the dominance degree of all the dimensionless parameters can be quantitatively evaluated and the dominant ones can be singled out conveniently. Taking a 1-D experiment of gas production from hydrate reservoir by depressurization as an example, we find that the order of sensitivity factors ranges from $10^{-5}$ to $10^{0}$. The most dominant dimensionless parameter is the dimensionless initial temperature of hydrate reservoir and the dimensionless phase equilibrium pressure of gas hydrate, which just reflects that the consumed energy for hydrate dissociation comes from the energy contained in formation and the driving force for hydrate dissociation is very important in hydrate dissociation by depressurization.
\end{abstract}

(c) 2012 Elsevier Ltd. All rights reserved.

\section{Introduction}

Gas hydrate is regarded as a kind of future energy due to its tremendous reserve [1] and widespread deposition [2]. With the continuous researching development on gas hydrate, the commercial production of gas hydrate is probably becoming a reality. Some theoretical and experimental works were carried out on how to exploit nature gas from hydrate reservoir by the safe, commercial and efficient methods. A lot of laboratory experiments have been carried out to study gas hydrate dissociation [3-8]. However few literatures have reported experiment based on the principle of similarity. The reasons may be that the hydrate dissociation process involves some complex mechanism which now cannot be clearly explained. Therefore, most experiments intended to reveal those kinds of unknown mechanisms. On the other hand, few institutes can obtain the nature samples of gas hydrate. Moreover, the measurement values for the same parameter may be with the difference of several orders which implies the lack of common view. Physical simulation obeying the principle of similarity is an important approach to reveal mechanism of gas hydrate dissociation which is helpful for filed design and optimization of development programs. Compared with field tests, physical simulation seems to be cheaper, time-saving and easier to implement.

\footnotetext{
* Corresponding author.

E-mail address: byh_2002@163.com (Y. Bai).
}

There are so many dimensionless parameters in physical simulation of hydrate reservoir exploitation because gas hydrate dissociation associates with such complicated factors as multiphase fluid flow in porous media, kinetic and endothermic process of hydrate dissociation, heat convection and conduction. According to the similarity of principle [9], each field prototype should theoretically exist a fully similar model which satisfies all the dimensionless parameters. However, it is very difficult or sometimes even impossible to keep all the dimensionless parameters identical in the laboratory experiment. To tackle this kind of problems, an efficient and practical way out is to single out the dominant parameters and to relax the secondary ones in laboratory experiments. Theoretical and experimental arranging all the dimensionless parameters in the orders of importance is not easy or even impossible to realize. In our work, a set of scaling criteria of gas production from hydrate reservoir by depressurization is firstly derived from the mathematical models. Then a numerical approach of sensitivity analysis of dimensionless parameters is proposed based on the scaling criteria of gas hydrates exploitation. With this approach, the dominance degree of the dimensionless parameters can be quantitatively evaluated and the dominant ones can be singled out conveniently.

\section{Sensitivity analysis of dimensionless parameters}

It is not realistic for us to estimate the role of each dimensionless parameter on experimental results by physical simulation, 
because too many dimensionless parameters are involved in gas hydrates exploitation experiment. Therefore, we propose a numerical approach to evaluate the effect of each dimensionless parameter on a target function. The sensitivity factor of a given dimensionless parameter $\pi_{i}$ is firstly defined as follows

$S_{i}=\frac{\partial\left(f / f_{p}\right)}{\partial\left(\pi_{i} / \pi_{i p}\right)}$

It denotes the relative variation ratio of a target function with respect to that of the dimensionless parameter $\pi_{i}$. Here $\pi_{i p}$ is the theoretical value of the given dimensionless parameter; $f$ is the target function of the physical model of gas hydrate reservoir which is defined as the function of all the dimensionless parameters and the dimensionless time $T_{D}$. The detail expression can be written as follows

$f=\int_{0}^{T} R\left(\pi_{1}, \pi_{2}, \ldots, \pi_{i}, \ldots, \pi_{n}, T_{D}\right) d T_{D}$

in which $R\left(\pi_{1}, \pi_{2}, \ldots, \pi_{i}, \ldots, \pi_{n}, T_{D}\right)$ is the dissociation ratio of gas hydrate defined as the ratio of dissociated gas hydrate to all hydrate bearing in formation. So the $f$ means the surrounding area of the axis of the dimensionless time and the dissociation ratio of gas hydrate as the dashed line shown in Fig. 1.

$f_{p}$ is the target function of the hydrate reservoir prototype and can be defined as

$f_{p}=\int_{0}^{T} R\left(\pi_{1}, \pi_{2}, \ldots, \pi_{i p}, \ldots, \pi_{n}, T_{D}\right) d T_{D}$

in which subscript $p$ denotes the gas hydrate reservoir prototype. So the $f_{p}$ denotes the surrounding area of the axis of the dimensionless time and the dissociation ratio of gas hydrate as the solid line shown in Fig. 1. In numerical scheme, the sensitivity factor can be written as in the following form

$S_{i}=\frac{\Delta f_{i} / f_{p}}{\left|w_{i}\right|}$

in which

$w_{i}=\frac{\pi_{i}-\pi_{i p}}{\pi_{i p}}$

representing for the relative distortion of the $i$ th dimensionless parameter.

$\Delta f_{i}=\int_{0}^{T}\left|R_{i}\left(\pi_{1}, \pi_{2}, \ldots, \pi_{i}, \ldots, \pi_{n}, T_{D}\right)-R_{p}\left(\pi_{1}, \pi_{2}, \ldots, \pi_{n}, T_{D}\right)\right| d t$ means the difference of dissociation ratio of gas hydrate between

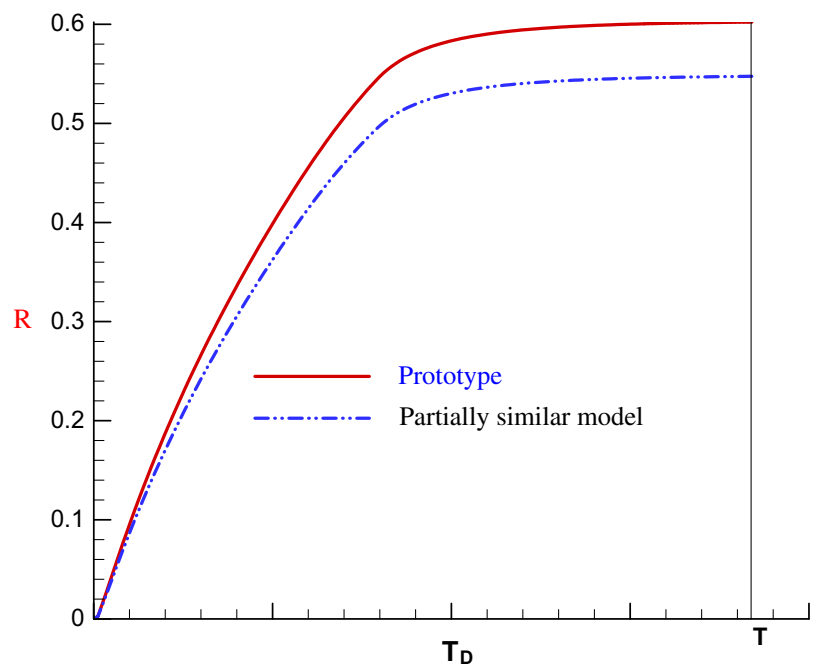

Fig. 1. The comparison of dissociation ratio of gas hydrate dissociation of prototype with a partially similar model, in which the ith dimensionless parameter is given a small deviation from the prototype. partially similar physical model that the $i$ th dimensionless parameter is relaxed and prototype.

\section{Mathematical mode}

Let us consider one-dimensional hydrate-bearing porous medium of length $L$. The production well is located at $x=0$, where the bottom pressure is fixed below the phase equilibrium pressure of gas hydrate. To reasonable simplify the physical problem some assumptions are introduced as follows: (1) gas hydrate in our assumed reservoir is methane hydrate; (2) water phase contains only water, gas phase contains only methane; (3) Darcy's law is valid for water and gas phases; and (4) the diffusion and the dispersion are neglected in mass transportation. With these concerned, the transport equations governing hydrate dissociation and the flow of gas and water in the porous media are

$\frac{\partial}{\partial x}\left(\frac{\rho_{g} k_{g}}{\mu_{g}} \frac{\partial p_{g}}{\partial x}\right)+\dot{m}_{g}=\frac{\partial\left(\phi \rho_{g} s_{g}\right)}{\partial t}$

$\frac{\partial}{\partial x}\left(\frac{\rho_{w} k_{w}}{\mu_{w}} \frac{\partial p_{w}}{\partial x}\right)+\dot{m}_{w}=\frac{\partial\left(\phi \rho_{w} s_{w}\right)}{\partial t}$

$-\dot{m}_{h}=\frac{\partial\left(\phi \rho_{h} s_{h}\right)}{\partial t}$

The energy conservative equation is written as

$$
\begin{aligned}
\frac{\partial}{\partial t}\left(C_{t} T\right)= & \frac{\partial}{\partial x}\left[\left(\rho_{w} C_{w} \frac{k_{w}}{\mu_{w}} \frac{\partial p_{w}}{\partial x}+\rho_{g} C_{p g} \frac{k_{g}}{\mu_{g}} \frac{\partial p_{g}}{\partial x}\right) T\right] \\
& +\frac{\partial}{\partial x}\left(K_{t} \frac{\partial T}{\partial x}\right)-\dot{m}_{h} \Delta H
\end{aligned}
$$

in which

$C_{t}=\phi\left(\rho_{w} s_{w} C_{w}+\rho_{g} s_{g} C_{V g}+\rho_{h} s_{h} C_{h}\right)+\rho_{r} C_{r}(1-\phi)$

$K_{t}=\phi s_{w} K_{w}+\phi s_{g} K_{g}+\phi s_{h} K_{h}+(1-\phi) K_{r}$

The relationship of saturations satisfy

$s_{w}+s_{g}+s_{h}=1$

The water and gas pressures are related by the following expression

$p_{c}=p_{w}-p_{g}$

The water and formation state equations are as follows when considering the slight compressibility.

$\rho_{w}=\rho_{w 0}\left(1+c_{w}\left(p_{w}-p_{w 0}\right)\right)$

$\phi=\phi_{0}\left(1+c_{\phi}\left(\frac{p_{w}+p_{g}}{2}-\frac{p_{w 0}+p_{g 0}}{2}\right)\right)$

With the boundary conditions

$x=0, \quad p=p_{g p}, \quad T=T_{g p}$

$x=L, \quad \frac{\partial p_{g}}{\partial x}=0, \quad \frac{\partial T}{\partial x}=0$

and the initial conditions

$p(x, 0)=p_{i}, \quad s_{h}(x, 0)=s_{h i}, \quad s_{w i}(x, 0)=s_{w i}, \quad T(x, 0)=T_{i}$

the governing equations are closed.

Here $\rho_{g}, \rho_{w}$ and $\rho_{h}$ are gas, water and hydrate densities, respectively; $\phi$ the porosity; $s_{g}, s_{w}$ and $s_{h}$ the saturations of gas, water and hydrate phases, respectively; $p_{w}$ and $p_{g}$ the water and gas pressures, respectively; $k_{\mathrm{g}}$ and $k_{w}$ the gas and water phase permeabilities, respectively; $\dot{m}_{h}, \dot{m}_{g}$ and $\dot{m}_{w}$ the mass rates of gas hydrate dissociation, gas and water production per unit formation volume, respectively; $C_{w}, C_{h}, C_{r}, C_{V g}$ and $C_{p g}$ the specific heats of water, hydrate, rock, the constant volume and pressure specific heats, 
respectively; $K_{w}, K_{g}, K_{r}$ and $K_{h}$ the coefficients of thermal conductivity of water, gas, rock and hydrate, respectively; $c_{w}$ and $c_{\phi}$ coefficients of compressibility of water and porous media, respectively; $p_{w 0}$ and $p_{g 0}$ the reference water and gas phase pressures, respectively; $\phi_{0}$ the porosity corresponding to $p_{w 0}$ and $p_{g 0}$; $p_{g p}$ and $T_{p}$ the pressure and temperature at the bottom of the production well, respectively; $p_{i}, s_{h i}, s_{w i}$ and $T_{i}$ the initial pressure, hydrate saturation, water saturation and temperature of the hydrate reservoir, respectively; $\Delta H$ is the enthalpy variation of hydrate dissociation.

\subsection{Gas hydrate dissociation kinetic model}

The Kim-Bishnoi model is adopted to evaluate the local mass rate of gas generated by hydrate dissociation as follows [10]

$\dot{m}_{g}=k_{d} A_{s}\left(f_{\text {eq }}-f_{g}\right)$

Here, $k_{d}$ is the dissociation kinetics constant; $f_{g}$ the local gas fugacity; $f_{\text {eq }}$ the gas equilibrium fugacity; $A_{s}$ is the specific surface area of porous media bearing gas hydrate and its variation relationship expression with time satisfies with Koznery-Carman model [11]

$A_{s}=\left(\phi_{e}^{3} / 2 k\right)^{0.5}$

in which $\phi_{e}$ is effective porosity and is defined as

$\phi_{e}=\phi\left(1-s_{h}\right)$

\subsection{The phase equilibrium model of gas hydrate}

The phase equilibrium pressure $p_{e q}$ is evaluated by [12]

$\log _{10} p_{e q}=a\left(T-T_{0}\right)+b\left(T-T_{0}\right)^{2}+c$

where $p_{e q}$ is in Pa and $T$ in Kelvin. $T_{0}$ is 273.15 K. $a, b, c$ are empirical constants depending on the hydrate composition. Using a least square fit [13], yields

$a=0.0342 K^{-1}, \quad b=0.0005 K^{-2}, \quad c=6.4804$

\subsection{Endothermic model of gas hydrate dissociation}

The process of hydrate dissociation is an endothermic phase change process. The latent heat for gas hydrate dissociation per kilogram of hydrate in $\mathrm{J} / \mathrm{kg}$ is given by [14]

$\Delta H=A T+B$

where $A, B$ are constants given by

$A=-1050 \mathrm{~J} /(\mathrm{kg} \mathrm{K}), \quad B=3,527,000 \mathrm{~J} / \mathrm{kg}$

\subsection{The state equation of gas}

The state equation of gas is

$p_{g} V=\frac{m}{M} Z R T$

where $Z$ is the gas deviation factor, $M$ the mass of the gas, $V$ the gas volume, $R$ the universal gas constant. Basing on Eq. (21), we obtain the gas compressibility $c_{g}$ as follows

$c_{g}=\frac{1}{\rho_{g}} \frac{d \rho_{g}}{d p_{g}}=\left(\frac{1}{p_{g}}-\frac{1}{Z} \frac{d Z}{d p_{g}}\right)$

For methane gas, in our concerning range of temperature and pressure, the compressibility is nearly proportional to the pressure [15], thus $\frac{d Z}{d p_{g}}=-7.5 \times 10^{-9}$

\subsection{Particular definition of some parameters of gas hydrate reservoir}

The pore space varies with hydrate saturation which leads to the variation of permeability of formation. Presently, no well known absolute permeability model is presented [16], the empirical relationship expression used by Yousif [17] is adopted based on the experimental results of Berea sandstone.

$k= \begin{cases}5.51721\left(\phi_{e}\right)^{0.86} & \phi_{e}<0.11 \\ 4.84653 \times 10^{8}\left(\phi_{e}\right)^{9.13} & \phi_{e} \geqslant 0.11\end{cases}$

In which the effective porosity is defined as $\phi_{e}(t)=\phi\left[1-s_{h}(t)\right]$, which implies the variation of the porosity space with time.

Note that, $s_{w}$ and $s_{g}$ solved from the governing equations are based on the total pore volume occupied by fluid phases and hydrate. However, when dealing with the capillary force and the relative permeability of water and gas phases, we revise water and gas saturations by defining them as effective water and gas saturations according to the following expressions

$s_{w}^{*}=s_{w} /\left(1-s_{h}\right)$

$s_{g}^{*}=s_{g} /\left(1-s_{h}\right)$

So, $s_{w}^{*}$ and $s_{g}^{*}$ denote the water and gas saturations based on effective pore volume, respectively.

\section{The scaling criteria of gas hydrate exploitation}

\subsection{The scaling criteria and their physical meaning}

To derive the scaling criteria, the dimensionless independent variables are first defined as follows

$x_{D}=\frac{x}{L}, \quad t_{D}=\frac{t}{T_{c}}=\frac{\dot{m}_{h \max } t}{s_{h i} \phi_{0} \rho_{h}}=\frac{p_{g p} t k_{d}}{s_{h i} \phi_{0} \rho_{h}} \sqrt{\frac{\phi_{0}^{3}}{2 k_{\max }}}$

in which $L$ is the characteristic length of hydrate reservoir.

The dimensionless dependent variables are defined as follows

$p_{g D}=\frac{p_{g}}{P_{g p}}, \quad p_{w D}=\frac{p_{w}}{P_{g p}}, \quad \bar{s}_{g}=\frac{s_{g}-s_{r g}}{1-s_{c w}-s_{r g}}=\frac{s_{g}-s_{r g}}{\Delta s}$

$\bar{s}_{w}=\frac{s_{w}-s_{c w}}{\Delta s}, \quad \bar{s}_{h}=\frac{s_{h}}{\Delta s}, \quad T_{D}=\frac{T}{T_{p}}$

So the scaling criteria for 1-D hydrate exploitation by depressurization can be derived from the governing equations by inspection analysis.

$$
\begin{aligned}
& \pi_{1}=\frac{k_{r g}}{k_{c w g}}, \quad \pi_{2}=\frac{k_{r w}}{k_{r g w}}, \quad \pi_{3}=\frac{k}{k_{\max }}, \quad \pi_{4}=\frac{\rho_{h}}{\rho_{g 0}}, \quad \pi_{5}=\frac{\rho_{r}}{\rho_{g 0}}, \\
& \pi_{6}=\frac{\rho_{w 0}}{\rho_{h}}, \quad \pi_{7}=\frac{K_{g}}{K_{w}}, \quad \pi_{8}=\frac{K_{h}}{K_{w}}, \quad \pi_{9}=\frac{K_{r}}{K_{w}}, \quad \pi_{10}=\frac{C_{v g}}{C_{w}}, \\
& \pi_{11}=\frac{C_{h}}{C_{w}}, \quad \pi_{12}=\frac{C_{r}}{C_{w}}, \quad \pi_{13}=\frac{\Delta H}{C_{w} T_{p}}, \quad \pi_{14}=\frac{p_{e q}}{p_{g p}}, \quad \pi_{15}=\frac{p_{g i}}{P_{g p}}, \\
& \pi_{16}=\frac{p_{w 0}}{p_{g p}}, \quad \pi_{17}=\frac{T_{i}}{T_{p}}, \quad \pi_{18}=\frac{s_{g i}-s_{r g}}{\Delta s}, \quad \pi_{19}=p_{g p} c_{w}, \\
& \pi_{20}=p_{g p} c_{\phi}, \quad \pi_{21}=s_{h i}, \quad \pi_{22}=s_{r g}, \quad \pi_{23}=s_{c w}, \quad \pi_{24}=\phi_{0}, \\
& \pi_{25}=\frac{k_{r g w} / \mu_{w}}{k_{c w g} / \mu_{g}}, \quad \pi_{26}=\frac{\rho_{g 0} k_{\max } k_{c w g}}{L^{2} \mu_{g} k_{d}} \sqrt{\frac{2 k_{\max }}{\phi_{0}^{3}}}, \\
& \pi_{27}=\frac{K_{h}}{L^{2} p_{g p} k_{d} C_{h}} \sqrt{\frac{2 k_{\max }}{\phi_{0}^{3}}}, \quad \pi_{28}=\frac{\sigma \cos \theta}{p_{g p}} \sqrt{\frac{\phi_{0}}{k_{\max }}}
\end{aligned}
$$


From the physical point of view, $\pi_{1}$ denotes the normalizing relative permeability of gas phase, namely, the ratio of the relative gas permeability under certain gas saturation to the relative gas permeability $k_{c w g}$ under irreducible water saturation; $\pi_{2}$ the normalizing relative permeability of water phase, namely, the ratio of the relative water permeability under certain water saturation to the relative water permeability $k_{\text {rgw }}$ under residual gas saturation; $\pi_{3}$ the dimensionless absolute permeability, in which $k_{\max }$ is the absolute permeability after hydrate fully dissociating; $\pi_{4}$ and $\pi_{5}$ the density ratios of gas hydrate and rock to natural gas under standard condition; $\pi_{6}$ the density ratio of water to gas hydrate; $\pi_{7}, \pi_{8}$ and $\pi_{9}$ the ratios of coefficients of thermal conduction of gas, hydrate and rock to water, respectively; $\pi_{10}, \pi_{11}$ and $\pi_{12}$ the ratios of specific heat of gas, hydrate and rock to water, respectively; $\pi_{13}$ the dimensionless latent heat of hydrate dissociation; $\pi_{14}, \pi_{15}$ and $\pi_{16}$ the ratios of phase equilibrium pressure of gas hydrate, initial gas pressure and reference pressure of water to the production pressure, respectively; $\pi_{17}$ the dimensionless initial temperature; $\pi_{18}$ the dimensionless initial gas saturation; $\pi_{19}$ and $\pi_{20}$ the volume variation ration of water and rock under the condition of bottom pressure of production well, respectively; $\pi_{21}$ the initial gas hydrate saturation; $\pi_{22}$ and $\pi_{23}$ the residual gas saturation and irreducible water saturation, respectively; $\pi_{24}$ the porosity after hydrate fully dissociating, namely the maximum porosity of formation; $\pi_{25}$ the ratio of the water mobility under residual gas saturation to the gas mobility under irreducible water saturation; $\pi_{26}$ the ratio of gas mass rate flowing through per unit area to gas mass rate produced by gas hydrate dissociation per unit area; $\pi_{27}$ the ratio of hydrate energy transferring by heat conduction to the energy contained in dissociated hydrate per unit time; $\pi_{28}$ the ratio of capillary pressure in average pore radius to the bottom pressure of the production well.

\subsection{The dimensionless governing equations}

The dimensionless governing equations containing aforementioned 28 dimensionless parameters can be written as follows.

The dimensionless mass balance equation of gas phase is given as

$$
\begin{aligned}
\pi_{26} & \frac{\partial}{\partial x_{D}}\left(\rho_{g D} \pi_{3} \pi_{1} \frac{\partial p_{g D}}{\partial x_{D}}\right)+\dot{m}_{g D} \\
= & \frac{1-\pi_{22}-\pi_{23}}{\pi_{21}} \frac{\partial}{\partial t_{D}}\left(\frac{\phi_{D} \rho_{g D} \bar{s}_{g}}{\pi_{4}}\right)+\frac{\pi_{22}}{\pi_{21}} \frac{\partial}{\partial t_{D}}\left(\frac{\phi_{D} \rho_{g D}}{\pi_{4}}\right)
\end{aligned}
$$

The dimensionless mass balance equation of water phase is given as

$$
\begin{gathered}
\frac{1-\pi_{22}-\pi_{23}}{\pi_{21}} \frac{\partial}{\partial t_{D}}\left(\phi_{D} \pi_{6} \rho_{w D} \bar{s}_{w}\right)+\frac{\pi_{23}}{\pi_{21}} \frac{\partial}{\partial t_{D}}\left(\phi_{D} \pi_{6} \rho_{w D}\right) \\
=\pi_{26} \pi_{25} \pi_{4} \pi_{6} \frac{\partial}{\partial x_{D}}\left(\rho_{w D} \pi_{3} \pi_{2} \frac{\partial p_{w D}}{\partial x_{D}}\right)+6.75 \dot{m}_{g D}
\end{gathered}
$$

The dimensionless mass balance equation of hydrate phase is given as

$$
-7.75 \dot{m}_{g D}=\frac{1-\pi_{22}-\pi_{23}}{\pi_{21}} \frac{\partial}{\partial t_{D}}\left(\phi_{D} \bar{s}_{h}\right)
$$

The dimensionless energy conservative equation is given as

$$
\begin{aligned}
\frac{\partial}{\partial t_{D}}\left(C_{t D} T_{D}\right)= & \pi_{26} \frac{\partial}{\partial x_{D}}\left(\rho_{g D} \pi_{3} \pi_{1} \pi_{10} \frac{\partial p_{g D}}{\partial x_{D}} T_{D}\right)+\pi_{25} \pi_{26} \pi_{4} \pi_{6} \\
& \times \frac{\partial}{\partial x_{D}}\left(\rho_{w D} \pi_{3} \pi_{2} \frac{\partial p_{w D}}{\partial x_{D}} T_{D}\right)+\frac{\partial}{\partial x_{D}}\left(K_{t D} \frac{\partial T_{D}}{\partial x_{D}}\right) \\
& -7.75 \dot{m}_{g D} \Delta H_{D}
\end{aligned}
$$

in which

$$
\begin{aligned}
\dot{m}_{g D}= & \sqrt{\frac{\left(1-\left(1-\pi_{22}-\pi_{23}\right) \bar{s}_{h}\right)^{3} \phi_{D}^{3}}{k_{D}}}\left(\pi_{14}-p_{g D}\right), \quad \rho_{g D}=\frac{Z_{0} T_{0} p_{g p}}{p_{0} Z T_{p}} \frac{p_{g D}}{T_{D}} \\
C_{t D}= & \frac{1-\pi_{22}-\pi_{23}}{\pi_{21}} \phi_{D} \frac{\pi_{6} \pi_{4} \rho_{w D} \bar{s}_{w}+\rho_{g D} \pi_{10} \bar{s}_{g}+\pi_{4} \pi_{11} \bar{s}_{h}}{\pi_{4}} \\
& +\frac{\pi_{23}}{\pi_{21}} \phi_{D} \pi_{6} \rho_{w D}+\frac{\pi_{22}}{\pi_{21}} \frac{\phi_{D} \rho_{g D} \pi_{10}}{\pi_{4}}+\frac{1}{\pi_{21} \pi_{24}} \frac{\pi_{5} \pi_{12}}{\pi_{4}}-\frac{1}{\pi_{21}} \\
& \times \frac{\pi_{5} \pi_{12} \phi_{D}}{\pi_{4}} \\
k_{t D}= & \frac{\pi_{27} \pi_{11} \pi_{24}}{\pi_{8}}\left(1-\pi_{23}-\pi_{23}\right) \phi_{D}\left(\bar{s}_{w}+\bar{s}_{g} \pi_{7}+\pi_{8} \bar{s}_{h}\right) \\
& +\frac{\pi_{27} \pi_{11} \pi_{24} \pi_{23}}{\pi_{8}} \phi_{D}+\frac{\pi_{27} \pi_{11} \pi_{24} \pi_{22}}{\pi_{8}} \phi_{D} \pi_{7}+\frac{\pi_{27} \pi_{11}}{\pi_{8}} \pi_{9} \\
& -\frac{\pi_{27} \pi_{11} \pi_{24}}{\pi_{8}} \phi_{D} \pi_{9}
\end{aligned}
$$

Here, $Z_{0}$ is the gas deviation factor, $p_{0}$ is the standard atmospheric pressure.

The dimensionless saturation relationship satisfies

$\bar{S}_{g}+\bar{S}_{w}=1$

The dimensionless state equations of water and formation are

$\rho_{w D}=1+\pi_{19}\left(p_{w D}-p_{w 0 D}\right)$

$\phi_{D}=1+\pi_{20}\left(p_{w D}-p_{w 0 D}\right)$

The dimensionless capillary pressure can be rewritten as

$p_{c D}=p_{g D}-p_{w D}=\pi_{28} \sqrt{\phi_{D} / \pi_{3}} J\left(\bar{s}_{w}\right)$

The dimensionless boundary condition satisfies

$x_{D}=0, \quad p_{g D}=1, \quad T_{D}=1$

$x_{D}=1, \quad \frac{\partial p_{g D}}{\partial x_{D}}=0, \quad \frac{\partial T_{D}}{\partial x_{D}}=0$

The dimensionless initial conditions are as follows

$p_{g D}\left(x_{D}, 0\right)=p_{g i D}, \quad \bar{s}_{h}\left(x_{D}, 0\right)=s_{h i}, \quad \bar{s}_{g}\left(x_{D}, 0\right)=\bar{s}_{g i}, \quad T_{D}\left(x_{D}, 0\right)=T_{i D}$

\subsection{The solution of dimensionless governing equations}

The governing equations are discretized using finite difference scheme with the convection term discretized using upwind scheme. hydrate saturation is firstly solved by explicit method. After the pressure is solved by implicit method the water saturation can be solved. Finally, the temperature is obtained by implicit method. The detail procedure is as follows.

The explicit discretized scheme of hydrate saturation is written as

$\bar{s}_{h i}^{n+1}=\bar{s}_{h}^{n}-\frac{\pi_{21} 7.75}{1-\pi_{22}-\pi_{23}} \frac{\dot{m}_{g_{D}} \Delta t_{D}}{\left(\phi_{D}\right)_{i}^{n}}$

where

$\dot{m}_{g D}=\sqrt{\frac{\left(1-\left(1-\pi_{22}-\pi_{23}\right) \bar{s}_{h}\right)^{3} \phi_{D}^{3}}{k_{D}}}\left(\pi_{14}-p_{g D}\right)$

The implicit discretized scheme of gas pressure is derived as

$a p_{g D i-1}+c_{c} p_{g D i}+b p_{g D i+1}=s_{p n}+s_{m}+s_{p c}+s_{p h}$ 
where

$a=a_{g D}+A a_{w D}, \quad b=b_{g D}+A b_{w D}, \quad c_{c}=c_{c g D}+A c_{c w D}, \quad s_{p n}=c_{c g D 2} p_{g D i}^{n}+A c_{c w D 2} p_{g D i}^{n}$

$s_{p c}=A\left(a_{w D} p_{c D i-1}+c_{c w D} p_{c D i}+b_{w D} p_{c D i+1}\right), s_{m}=-\dot{m}_{g D}-6.75 A \dot{m}_{g D}$

$A=\frac{\rho_{g D i}}{\pi_{4} \pi_{6} \rho_{w D i}}, s_{p h}=\frac{1-\pi_{23}-\pi_{24}}{\pi_{22}} \frac{\phi_{D i} \rho_{g D i}}{\pi_{4}} \frac{\bar{s}_{h}^{n}-\bar{s}_{h}^{n+1}}{\Delta t_{D}}$

$a_{g D}=\frac{\pi_{26}\left(\rho_{g D} \pi_{3} \pi_{11}\right)_{i-\frac{1}{2}}}{x_{D i}^{2}}, a_{w D}=\pi_{26} \pi_{25} \pi_{4} \pi_{6} \frac{\left(\rho_{w D} \pi_{3} \pi_{2}\right)_{i-\frac{1}{2}}}{x_{D i}^{2}}$

$b_{g D}=\frac{\pi_{26}\left(\rho_{g D} \pi_{3} \pi_{1}\right)_{i+\frac{1}{2}}}{\Delta x_{D i}^{2}}, b_{w D}=\pi_{26} \pi_{25} \pi_{4} \pi_{6} \frac{\left(\rho_{w D} \pi_{3} \pi_{2}\right)_{i+\frac{1}{2}}}{\Delta x_{D i}^{2}}$

$c_{c g D}=c_{c g D 1}+c_{c g D 2}, c_{c g D 1}=-\left(a_{g D}+b_{g D}\right)$

$c_{c g D 2}=-\left(\phi_{D i}\left(\frac{\partial \rho_{g D}}{\partial p_{g D}}\right)_{i}+\rho_{g D i} \pi_{20}\right) \frac{1}{\rho_{h D}}\left(\frac{1-\pi_{22}-\pi_{23}}{\pi_{21}} \bar{s}_{g i}+\frac{\pi_{22}}{\pi_{21}}\right) \frac{1}{\Delta t_{D}}$

$c_{c w D}=c_{c w D 1}+c_{c w D 2}, \quad c_{c w D 1}=-\left(a_{w D}+b_{w D}\right)$,

$c_{c w D 2}=-\pi_{6}\left(\phi_{D i} \pi_{19}+\rho_{w D i} \pi_{20}\right)\left(\frac{1-\pi_{22}-\pi_{23}}{\pi_{21}} \bar{s}_{w i}+\frac{\pi_{23}}{\pi_{21}}\right) \frac{1}{\Delta t_{D}}$

The implicit discretized scheme of energy conservative equation is derived as

$$
\begin{aligned}
& \left(k_{B D}+k_{D D}\right) T_{D i+1}-\left(k_{C D}+k_{D D}+k_{A D}+\frac{c_{t D i}^{n+1}}{\Delta t_{D}}\right) T_{D i}+k_{C D} T_{D i-1} \\
& =-\frac{c_{t D i}^{n}}{\Delta t_{D}} T_{D i}^{n}+7.75 \dot{m}_{g D} \Delta H_{D}
\end{aligned}
$$

where

$$
\begin{aligned}
k_{A D}= & \pi_{27} \frac{\left(\rho_{g D} \pi_{1} \pi_{3} C_{v g D}\right)_{i-\frac{1}{2}}\left(p_{g D i}-p_{g D i-1}\right)}{\Delta x_{D i}^{2}} \\
& +\pi_{26} \pi_{25} \pi_{4} \pi_{6} \frac{\left(\rho_{w D} \pi_{1} \pi_{3}\right)_{i-\frac{1}{2}}\left(p_{w D i}-p_{w D i-1}\right)}{\Delta x_{D i}^{2}} \\
k_{B D}= & \pi_{27} \frac{\left(\rho_{g D} \pi_{1} \pi_{2} C_{v g D}\right)_{i+\frac{1}{2}}\left(p_{g D i+1}-p_{g D i}\right)}{\Delta x_{D i}^{2}} \\
& +\pi_{26} \pi_{25} \pi_{4} \pi_{6} \frac{\left(\rho_{w D} \pi_{1} \pi_{3}\right)_{i+\frac{1}{2}}\left(p_{w D i}-p_{w D i+1}\right)}{\Delta x_{D i}^{2}} \\
k_{C D}= & \frac{K_{t D i-\frac{1}{2}}}{\Delta x_{D i}^{2}}, k_{D D}=\frac{K_{t D i+\frac{1}{2}}}{\Delta x_{D i}^{2}}
\end{aligned}
$$

To verify the mathematical model and numerical solution, the numerical results of 1-D hydrate dissociation systems by depressurization were compared with experimental results obtained by us in Guangzhou Center for Gas Hydrate Research, Chinese Academy of Sciences. These experiment systems consist of such seven parts as the stable liquid and gas supplying, the hydrate formation and dissociation vessel, the environment simulation, the backpressure regulator controlling, computation and data acquisition. The detail description of experimental system and procedures can be found in the literature[18]. The main physical variable values used in 1-D model in numerical simulation are listed as Table 1 in which the values were determined by experiment. Figs. 2 and 3 show the comparisons of cumulative gas volumes and pressures of numerical and experimental results for 1-D gas hydrate dissociation system under depressurization condition. We can see that the

Table 1

Main physical variable values determined by experiment.

\begin{tabular}{ll}
\hline Physical variable values & 1 -D system \\
\hline Absolute permeability $(\mathrm{d})$ & 1.11 \\
Porosity & 0.328 \\
Initial pressure $(\mathrm{MPa})$ & 3.584 \\
Initial temperature $\left({ }^{\circ} \mathrm{C}\right)$ & 2.08 \\
Backpressure $(\mathrm{MPa})$ & 1.94 \\
Initial water saturation & 0.193 \\
Initial hydrate saturation & 0.254 \\
\hline
\end{tabular}

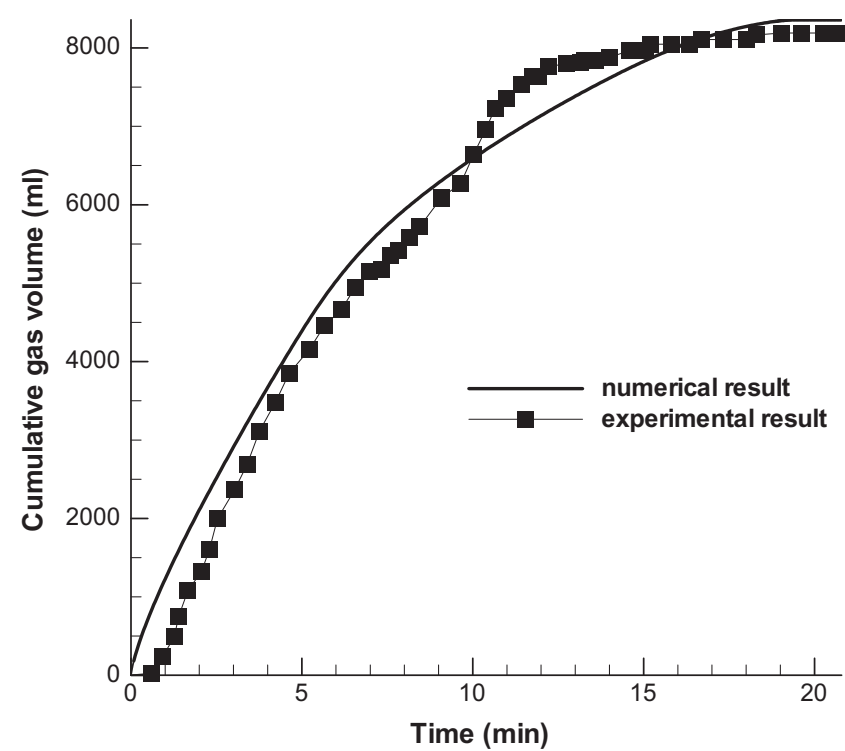

Fig. 2. Comparison of cumulative gas volumes of numerical and experimental results for 1-D model.

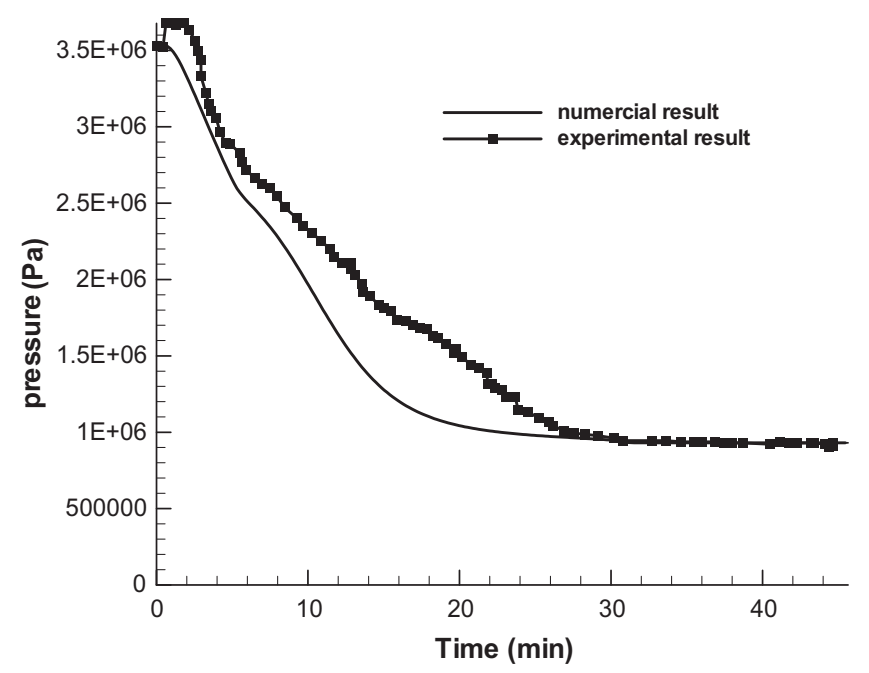

Fig. 3. Comparison of pressures of numerical and experimental results for 1-D model.

numerical results agree well with the experiment which completes the validation of the mathematical model and numerical code employed.

\section{Results and discussion}

The values of main physical variables in hydrate reservoir prototype are listed in Table 2 . Then the values of all dimensionless parameters can be calculated as follows.

$\pi_{4}=1260.5, \quad \pi_{5}=3501.4, \quad \pi_{6}=1.11, \quad \pi_{7}=0.125$,

$\pi_{8}=6.07, \quad \pi_{9}=5.36, \quad \pi_{10}=0.524, \quad \pi_{11}=0.427$,

$\pi_{12}=0.199, \quad \pi_{15}=5.2, \quad \pi_{16}=5.2, \quad \pi_{17}=1, \quad \pi_{18}=0.126$,

$\pi_{19}=1.25 \times 10^{-3}, \quad \pi_{20}=2 \times 10^{-3}, \quad \pi_{21}=0.62, \quad \pi_{22}=0.035$,

$\pi_{23}=0.256, \quad \pi_{24}=0.1, \quad \pi_{25}=9.32 \times 10^{-3}, \quad \pi_{26}=1.0172 \times 10^{-4}$,

$\pi_{27}=1.143 \times 10^{-5}, \quad \pi_{28}=0.0374$

The others are dimensionless functions. Set $1 \%$ variation for each dimensionless parameter and keep the others identical between the partially similar model and the prototype. The 
Table 2

The values of the main physical variables.

\begin{tabular}{llll}
\hline Variables & Values & Variables & Values \\
\hline$p_{i}(\mathrm{MPa})$ & 13 & $C_{r}(\mathrm{~J} / \mathrm{K} \mathrm{kg})$ & 840 \\
$p_{g p}(\mathrm{MPa})$ & 2.5 & $K_{g}(\mathrm{~W} / \mathrm{m} \mathrm{K})$ & 0.07 \\
$T_{g p}(\mathrm{~K})$ & 288 & $K_{h}(\mathrm{~W} / \mathrm{m} \mathrm{K})$ & 3.4 \\
$T_{i}(\mathrm{~K})$ & 288 & $K_{w}(\mathrm{~W} / \mathrm{m} \mathrm{K})$ & 0.56 \\
$s_{w i}$ & 0.257 & $K_{r}(\mathrm{~W} / \mathrm{m} \mathrm{K})$ & 3 \\
$s_{h i}$ & $\rho_{w}\left(\mathrm{~kg} / \mathrm{m}^{3}\right)$ & 1000 \\
$k_{d}\left(\mathrm{~kg} / \mathrm{m}^{2}\right.$ Pa s $)$ & $4.4 \times 10^{-16}$ & $\rho_{h}\left(\mathrm{~kg} / \mathrm{m}^{3}\right)$ & 910 \\
$C_{v g}(\mathrm{~J} / \mathrm{K} \mathrm{kg})$ & 2206 & $\rho_{r}\left(\mathrm{~kg} / \mathrm{m}^{3}\right)$ & 2500 \\
$C_{w}(\mathrm{~J} / \mathrm{K} \mathrm{kg})$ & 4211 & $\phi_{0}(\%)$ & 18.8 \\
$C_{h}(\mathrm{~J} / \mathrm{K} \mathrm{kg})$ & 1800 & & \\
\hline
\end{tabular}

sensitivity factor of each dimensionless parameter can be calculated and shown in Fig. 4.

We can see from Fig. 4 that the order of sensitivity factor ranges from $10^{-5}$ to $10^{0}$. Apparently, the larger the sensitivity factor, the more important of the corresponding dimensionless parameter. The most sensitive dimensionless parameters with the sensitivity factor order around $10^{\circ}$ are

$\pi_{17}=\frac{T_{i}}{T_{p}}, \quad \pi_{14}=\frac{p_{e q}}{p_{g p}}$

The more sensitive dimensionless parameters which sensitivity factor order are around $10^{-1}$ are as follows

$\pi_{1}=\frac{k_{r g}}{k_{c w g}}, \quad \pi_{3}=\frac{k}{k_{\max }}, \quad \pi_{4}=\frac{\rho_{h}}{\rho_{g 0}}, \quad \pi_{5}=\frac{\rho_{r}}{\rho_{g 0}}, \quad \pi_{6}=\frac{\rho_{w 0}}{\rho_{h}}$,

$\pi_{12}=\frac{C_{r}}{C_{w}}, \quad \pi_{13}=\frac{\Delta H}{C_{w} T_{p}}, \quad \pi_{15}=\frac{p_{g i}}{P_{g p}}, \quad \pi_{21}=s_{h i}, \quad \pi_{23}=s_{c w}$,

$\pi_{24}=\phi_{0}, \quad \pi_{26}=\frac{\rho_{g 0} k_{\max } k_{c w g}}{L^{2} \mu_{g} k_{d}} \sqrt{\frac{2 k_{\max }}{\phi_{0}^{3}}}$

Actually, the most dominant dimensionless parameter is the dimensionless initial temperature of the hydrate reservoir. The reason can be that the energy consumed by hydrate dissociation in depressurization mainly comes from the formation and the surroundings because of no other thermal source in the hydrate reservoir. Therefore, the initial temperature of the hydrate reservoir is dominant factor to control the amount of hydrate dissociation. The dimensionless phase equilibrium pressure is also the dominant one because the driving force of hydrate dissociation is the difference between the phase equilibrium pressure and gas pressure. Thus, the phase equilibrium pressure exerts more effect on hydrate dissociation. These two dimensionless parameters just reflect the principle of gas production from hydrate reservoir by depressurization. Moreover, normalizing relative permeability of gas phase, dimensionless absolute permeability of formation, the density ratios of hydrate and rock to gas, the density ratio of water to hydrate, the specific heat ratio of rock to water, the dimensionless latent heat of hydrate dissociation, the dimensionless initial gas pressure and hydrate saturation, irreducible water saturation and the ratio of gas mass rate flowing through per unit area to gas mass rate produced by gas hydrate dissociation per unit area are relative dominant ones. The dominance degree of these dimensionless parameters implies that the parameters such as hydrate density, latent heat, phase equilibrium pressure and initial hydrate saturation are relative dominant for gas hydrate. For gas phase, the gas relative permeability, initial gas pressure and the ratio of gas mass rate flowing per unit area to gas mass rate produced by gas hydrate dissociation per unit area are relative dominant. For water phase, such parameters as irreducible water saturation and water density are relative important; For rock, such parameters as absolute permeability, density, specific heat and porosity are important. So in laboratory experiment, these dominant ones should be satisfied firstly when they are contradictory with other secondary dimensionless parameters.

\section{Conclusions}

There are too many dimensionless parameters for hydrate exploitation experiment to keep all dimensionless parameters identical in one experiment. Therefore, we must know which one is dominant that should be satisfied with and which one is the secondary that can be relaxed. In our research work, we derive a set of scaling criteria for hydrate exploitation physical simulation. A numerical approach of sensitivity analysis of dimensionless parameters is proposed based on the analysis of the sensitivity factor defined as the relative variation ration of a target function with respect to the relative variation of dimensionless parameters. We find that the order of sensitivity factors ranges from $10^{-5}$ to $10^{0}$. With this approach, the dominance degree of the dimensionless parameters can be quantitatively evaluated and the dominant ones

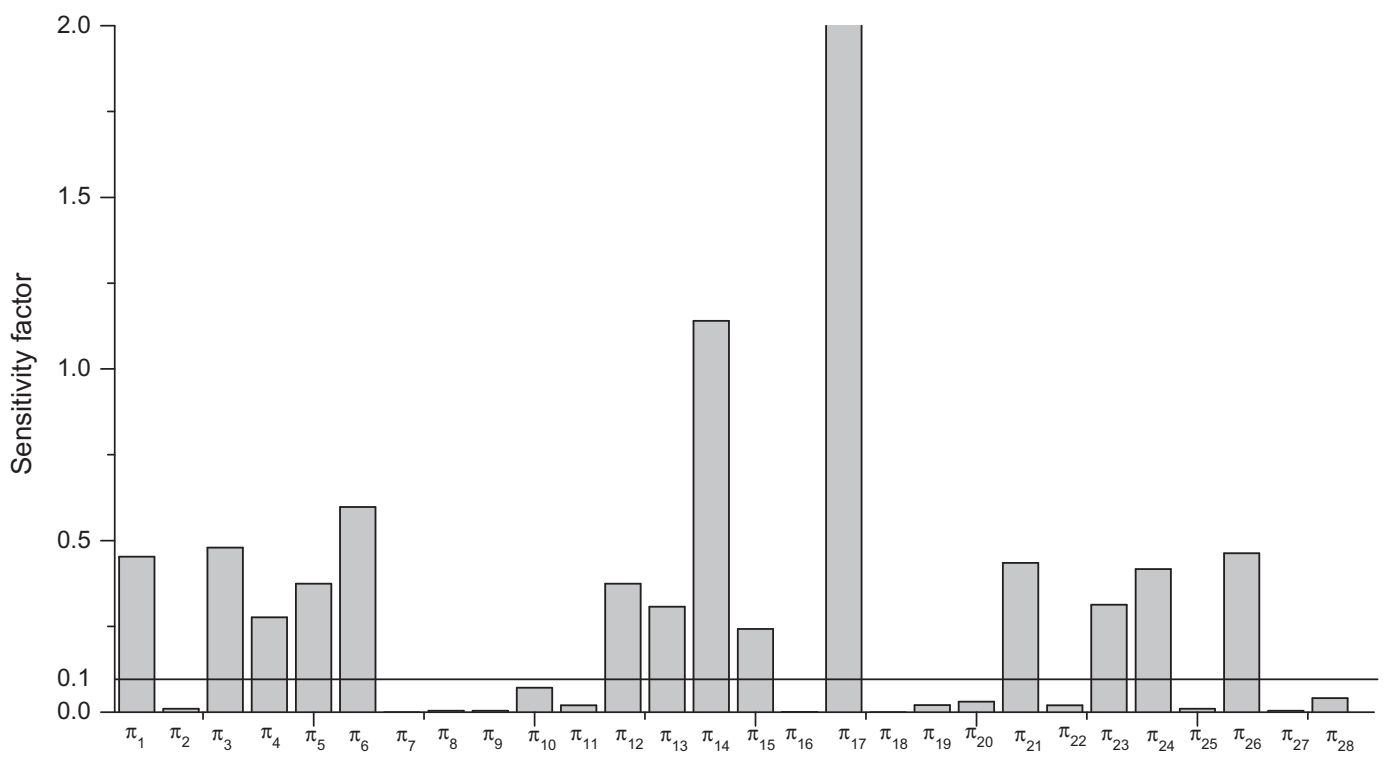

Fig. 4. The comparison of sensitivity factors of all the dimensionless parameters. 
can be singled out conveniently. The most dominant dimensionless parameter is the dimensionless initial temperature of the hydrate reservoir and the dimensionless phase equilibrium pressure of gas hydrate, which just reflects that the consumed energy for hydrate dissociation comes from the energy contained in formation and the driving force for hydrate dissociation is very important in hydrate dissociation by depressurization.

\section{Acknowledgement}

This work is financially supported by the Zhejiang Provincial Natural Science Foundation of China (Y4110245).

\section{References}

[1] Ayhan D. Methane hydrates as potential energy resource: Part 1 - Importance, resource and recovery facilities. Energy Convers Manage 2010;51(7):1547-61.

[2] Kvenvolden KA. A primer on the geological occurrence of gas hydrate. Geol Soc Lond 1998;Spec. Publ. 137:9-30.

[3] Akihiro H, Ryo N, Daisuke $\mathrm{K}$, et al. Dissociation heat of mixed-gas hydrate composed of methane and ethane. In: Proceedings of the 6th international conference on gas hydrate (ICGH 2008), Vancouver, British Columbia, Canada; July $6-10,2008$.

[4] Taro K, Michika O, Yasuhide S, et al. Experimental study of enhanced gas recovery from gas hydrate bearing sediments by inhibitor and steam injection methods. In: Proceedings of the 6th international conference on gas hydrate (ICGH 2008), Vancouver, British Columbia, Canada; July 6-10, 2008.

[5] Yang X, Sun CY, Su KH, et al. A three-dimensional study on the formation and dissociation of methane hydrate in porous sediment by depressurization. Energy Convers Manage 2012;56:1-7.
[6] Lee J, Park S, Sung W. An experimental study on the productivity of dissociated gas from gas hydrate by depressurization scheme. Energy Convers Manage 2010;51(12):2510-5.

[7] Bahman ZN, Farshad V. A generalized macroscopic kinetic model for description of gas hydrate formation processes in isothermal-isochoric systems. Energy Convers Manage 2012;57:125-30.

[8] Komai T, Sakamoto Y, Kawamura T, Yamamoto Y. Dissociation rate of methane hydrates occupied in pore space of marine sediments. In: Proceeding of the sixth (2005) ISOPE ocean mining symposium, Changsha, Hunan, China; October 9-13, 2005.

[9] Sedov LI. Similarity and dimensional methods in mechanics. Academic Press; 1959.

[10] Kim HC, Bishnoi PR, Heidemann RA, Rizvi SSH. Kinetics of methane hydrate dissociation. Chem Eng Sci 1987;42(7):1645-53.

[11] Amyx HC, Bass DM, Whiting RL. Petroleum reservoir engineering physical properties. New York City: McGraw-Hill Book Co.; 1960.

[12] Makogon YF. Hydrate of hydrocarbons. Oklahoma: PennWell Publishing Co. Tulsa; 1997.

[13] Ji C, Ahmadi G, Smith DH. Natural gas production form hydrate decomposition by depressurization. Chem Eng Sci 2001;56:5801-14.

[14] Kamath V. Study of heat transfer characteristics during dissociation of gas hydrate in porous media. PhD thesis, University of Pittsburgh, Pittsburgh; 1983.

[15] Hong SD. The principle of reservoir physics. Beijing: Petroleum Industry Press; 1985.

[16] Mehran PD. Gas production from hydrate reservoirs and its modeling. SPE 86827; 2004.

[17] Yousif MH, Abass HH, Selim MS, Sloan ED. Experimental and theoretical investigation of methane-gas-hydrate dissociation in porous media. SPE 18320; 1991. p. 69-76.

[18] Tang LG, Li XS. Control mechanisms for gas hydrate production by depressurization in different scale hydrate reservoirs. Energy Fuel 2007;21(1):227-33. 